\title{
Phytochemical diversity and antimicrobial properties of methanol extract of several cultivars of Catharanthus roseus using GC-MS
}

\author{
SITI SAMIYARSIH`, NUR FITRIANTO, ELLY PROKLAMASININGSIH, JUWARNO, \\ JUNI SAFITRI MULJOWATI \\ Faculty of Biology, Universitas Jenderal Soedirman. Jl. Dr. Suparno No. 63, Grendeng, Purwokerto, Banyumas 53122, Central Java, Indonesia. \\ Tel.: +62-281-638794, Fax. +62-281-631700, ^email: asih.fbio@gmail.com
}

Manuscript received: 27 November 2019. Revision accepted: 7 March 2020

\begin{abstract}
Samiyarsih S, Fitrianto N, Proklamasiningsih E, Juwarno, Muljowati JS. 2020. Phytochemical diversity and antimicrobial properties of methanol extract of several cultivars of Catharanthus roseus using GC-MS. Biodiversitas 21: 1332-1344. Catharanthus roseus (L.) $\mathrm{G}$ is an important medicinal plant to evaluate the possibility of novel pharmaceuticals since most of the bacterial pathogens are developing resistance against antibiotics. This research aimed to determine the phytochemical diversity of methanol extract of eight cultivars of $C$. roseus and to evaluate for possible antimicrobial (antifungal and antibacterial) activities. It is the first research to compare phytochemicals and antimicrobial potential among C. roseus cultivars. The compound obtained was screened by Gas ChromatographyMass Spectrometry (GC-MS) method. While agar-well disc diffusion method was employed to measure antimicrobial activity against Escherichia coli, Staphylococcus aureus, Aspergillus flavus and Aspergillus niger. Based on GC-MS analysis, a total of 18 significantly different metabolite compounds. The abundances of phytochemical compounds (18 classes total) in each cultivar were Dark Pink (66\%), Pink (50\%), Purple Pink (44\%), Pale Pink (27\%), White (44\%), Milky White (50\%), Whitish pink (50\%) and Pinkish Red (55\%). The leaves extracts showed antimicrobial activity with inhibition zones ranging from 6.40-22.00 mm and 3.35-8.20 mm, respectively. The best antimicrobial activity against $E$. coli, $S$. aureus, A. flavus, and A. niger with the zone of inhibition $16.10 \pm 1.67 \mathrm{~mm}, 22.00 \pm 0.33 \mathrm{~mm}$, $6.05 \pm 0.67 \mathrm{~mm}$ and $8.20 \pm 0.50 \mathrm{~mm}$ respectively by Dark Pink cultivar.
\end{abstract}

Keywords: Antimicrobial, Catharanthus roseus, GC-MS, phytochemical

\section{INTRODUCTION}

Catharanthus roseus (L.) G. Don is an important medicinal plant of the family Apocynaceae. This plant found to be rich in their pharmacological actions that include antibacterial, antifungal, antioxidant, anticancer, and antiviral activates (Gajalakshmi et al. 2013). C. roseus substances are the basis for the use of treatment of human diseases and the development of new drugs (Almagro et al. 2015).

The diversity of $C$. roseus cultivars is determined based on the morphology and the color of corolla. The existence of the $C$. roseus cultivars is a precious asset as part of germplasm conservation (Adnan et al. 2016). Different cultivars have different responses to the phytochemical content and biological activity (Lal et al. 2011; El-Domyati et al. 2012). In Indonesia, this plant has traditionally used to treat malaria, constipation, diuretics, diabetes mellitus, and hypertension (Pandiangan et al. 2013). C. roseus leaves were many used as alkaloidproducing medicinal plants. A detailed literature survey about the medicinal properties of $C$. roseus leaves revealed it to possess antileukemic, antioxidant, antimicrobial, and anti-inflammatory activities (Jacobs et al. 2004). Recent studies have shown other chemical compounds in this plant with strong antiradical activity such as new phenolic compounds in the seed, stem, leaf, and the petal, and organic acids in the root (Pereira et al. 2010). The extracts of many organs of this plant also exhibit antimicrobial effects (Patil and Ghosh 2010).

Methanolic extracts from different parts of $C$. roseus have tested for antimicrobial activity. Notably, the leaf extracts showed significantly higher activity, suggesting that bioactive compounds of $C$. roseus can be used as an antimicrobial agent (Nayak and Pereira 2006). Due to the importance of these essential secondary metabolites, numerous studies demonstrate the antiviral and antimicrobial activities of the alkaloids obtained from $C$. roseus (Koul et al. 2013). Pathogenic gram-negative bacteria like $E$. coli and gram-positive bacteria like $S$. aureus are zoonotic bacteria. The emergence of antimicrobial-resistant strains becomes a critical issue in both human and animal health globally. The resistance of $S$. aureus to antimicrobials is a considerably growing concern, with the overall rates varying widely by region (Mekonnen et al. 2018). S. aureus is the most common cause of hospital-acquired wound infections (Atef et al. 2019). A. flavus is one of the most common causes of otomycosis (fungal ear infections), which can cause pain, temporary hearing loss, and, in severe cases, damage to the ear canal and tympanic membrane. A. niger causes chronic pulmonary infections or allergic disease in immunecompetent hosts (Chaman et al. 2013).

The preliminary phytochemical screening of different extracts carried out in the presence of various phytoconstituents, such as alkaloids, carbohydrates, 
glycosides, flavonoids, tannins, and phenolic compound (Kar et al. 2018). Phytochemical screening of the methanol extract of $C$. roseus disclosed the presence of alkaloids, terpenoids, phenols, tannins, saponins, quinines, flavonoids, and proteins. Identified and confirmed the structures of the constituents present utilizing a hyphenated technique of gas chromatography-mass spectrometry (GCMS) from the extract (Doshi et al. 2018). The most attractive values of $C$. roseus can produce more than 130 kinds of terpenoid indole alkaloids (TIAs) (Chen et al. 2017).

Gas chromatography-mass spectrometry (GC-MS) has emerged as a promising method for the analysis of bioactive constituents. It has employed to identify the various bioactive components, e.g., non-polar compounds, long-chain hydrocarbons, alcohols, acids, esters, alkaloids, amino and nitro compounds with volatile oil, fatty acids, and lipids (Velmurugan and Anand 2017). The research aimed to determine the phytochemical profile of methanol extract from eight cultivars of $C$. roseus using GC-MS analysis and to evaluate for antimicrobial (antifungal and antibacterial) activities.

\section{MATERIALS AND METHODS}

\section{Study area}

The research employed a survey method, with purposive sampling in the Banyumas District, Central Java, Indonesia as shown on the following location map (Figure 1).

\section{Procedures}

Plant materials

Catharanthus roseus plants were taken in several areas in Banyumas District, including Sub-districts of Kemranjen, Banyumas, Sokaraja, Sumbang, Baturraden, and Ajibarang (Figure 1; Table 1). Living plants of eight cultivars of $C$. roseus collected in 28 sites during the field survey. The plant seeds $C$. roseus collected from several places were planted in the greenhouse of the Faculty of Biology Jenderal Soedirman University for three months. Plants were plantlet under controlled conditions at a temperature of $30 / 27^{\circ} \mathrm{C}$ day/night, and relative humidity of $65 \%$. After three months of planting, the leaves of each cultivar extracted to be analyzed for phytochemical content by GC-MS and antimicrobial properties.

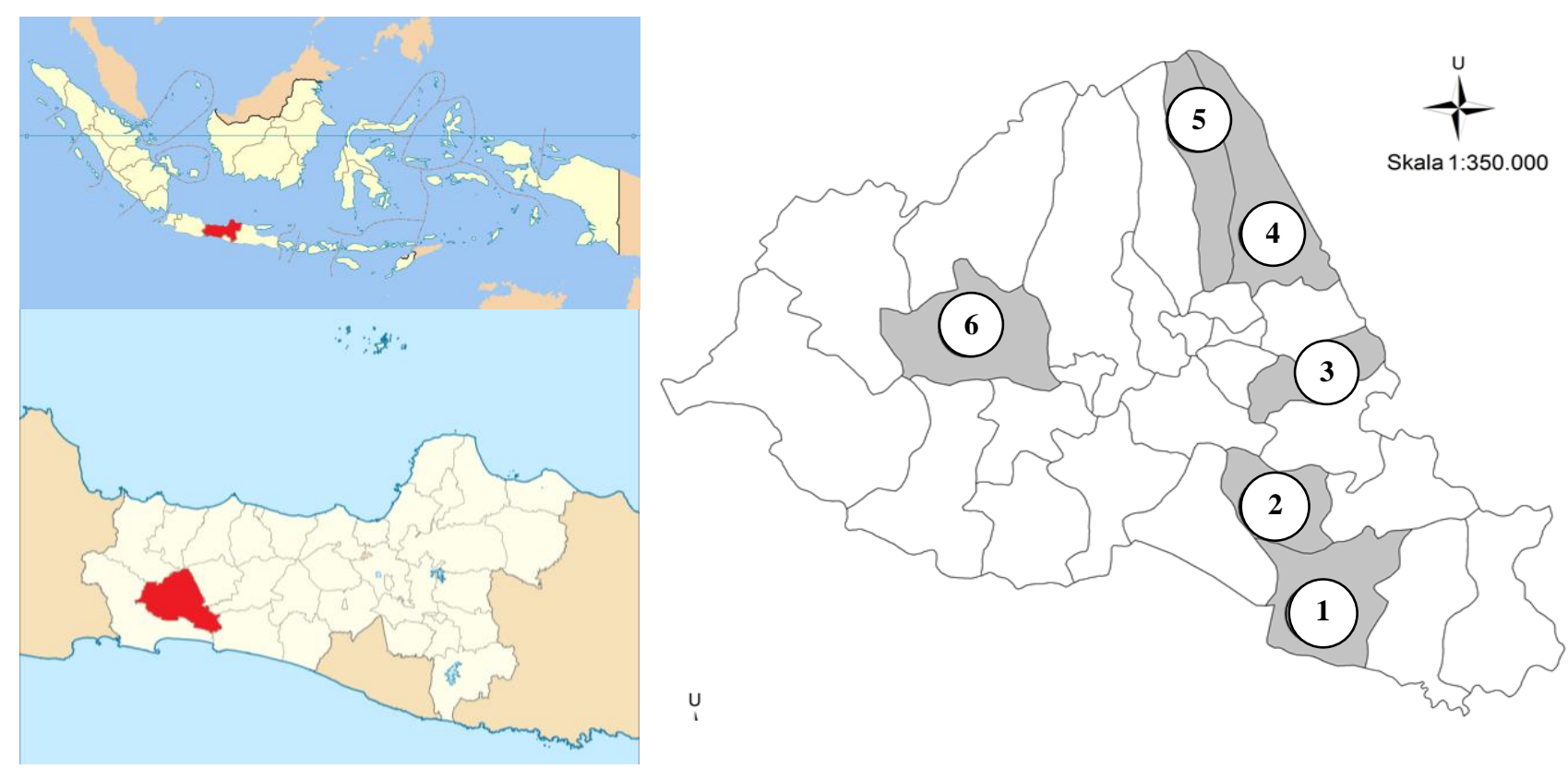

Figure 1. The selected sampling sites map of the Catharanthus roseus area in Banyumas District, Central Java, Indonesia: 1. Subdistrict Kemranjen, 2. Sub-district Banyumas, 3. Sub-district Sokaraja, 4. Sub-district Sumbang, 5. Sub-district Baturraden, 6. Sub-district Ajibarang. Detailed the sample site and status of eight cultivars of $C$, roseus is presented in Table 1.

Table 1. The sample site and status of Catharanthus roseus studied in Banyumas District, Central Java, Indonesia

\begin{tabular}{lllcc}
\hline Sample site (village, sub-district) & Cultivar & Status & Coordinate & Altitude (m asl.) \\
\hline Karangsalam, Kemranjen & White & Wild & $7^{\circ} 33^{\prime} 51.5^{\prime \prime} \mathrm{S} 109^{\circ} 20^{\prime} 11.2^{\prime \prime} \mathrm{E}$ & 548 \\
Papringan, Banyumas & Milky white & Wild & $7^{\circ} 29^{\prime} 58.2^{\prime \prime} \mathrm{S} 109^{\circ} 14^{\prime} 25.9^{\prime \prime} \mathrm{E}$ & 140 \\
Sokaraja Lor, Sokaraja & Whitish pink & Wild & $7^{\circ} 25^{\prime} 49.7^{\prime \prime} \mathrm{S} 109^{\circ} 19^{\prime} 13.7^{\prime \prime} \mathrm{E}$ & 36 \\
Banteran, Sumbang & Pinkish red & Cultivated & $7^{\circ} 21^{\prime} 39.9^{\prime \prime} \mathrm{S} 109^{\circ} 15^{\prime} 59.1^{\prime \prime} \mathrm{E}$ & 225 \\
Kemutug Lor, Baturraden & Dark Pink, Purple Blue & Cultivated & $7^{\circ} 15^{\prime} 34.7^{\prime \prime} \mathrm{S} 109^{\circ} 12^{\prime} 50.2^{\prime \prime} \mathrm{E}$ & 650 \\
Ciberung, Ajibarang & Pink, Pale Pink & Wild & $7^{\circ} 23^{\prime} 13.3^{\prime \prime} \mathrm{S} 109^{\circ} 03^{\prime} 48.4^{\prime \prime} \mathrm{E}$ & 227 \\
\hline
\end{tabular}


Sample collection and preparation of leaf extract C. roseus

Fresh leaves were collected and thoroughly washed with distilled water and dried at $37^{\circ} \mathrm{C}$. Then, the dried leaves were ground to make a fine powder using mortar and pestle. C. roseus leaf extraction (old leaves/ generative phase) was carried out by the maceration method. The maceration method is the extraction technique of samples that were still solid or still concentrated using certain solvents (Sasongko and Samiyarsih 2018). Leaves washed thoroughly using running water, then dried in powder form. The dried powder was macerated with $100 \mathrm{~mL}$ methanol and stored for 48 hours in an oven at $60^{\circ} \mathrm{C}$. After 48 hours, the extract was dissolved in methanol, then filtered with Whatman filter paper No. $42(125 \mathrm{~mm})$, and the organic layer is allowed to evaporate. The resulting black, green extract concentrated using a rotary evaporator with a water bath at $40^{\circ} \mathrm{C}$. The crude extract was lyophilized into a paste and used for further analysis (Vanitha et al. 2011).

\section{Phytochemical component by Gas Chromatography-Mass Spectrum Analysis (GC-MS)}

Identification of phytochemical components of $C$. roseus methanol leaf extract was carried out by GC-MS analysis (GC-MS QP 2010 Ultra, Shimadzu) at a concentration of $10 \mathrm{mg} / \mathrm{mL}$ with methanol solvent. Five $\mu \mathrm{L}$ samples $(10 \mathrm{mg} / \mathrm{ml}$ in methanol) injected into the RTX-5ms column (Restek, USA) (0.25um thick, $0.25 \mathrm{~mm}$ diameter, and $30 \mathrm{~m}$ length by using, autocomplete AOC-20S (Shimadzu) and autoinjector AOC-20I. The analysis conducted at the Integrated Laboratory of Muhammadiyah University Purwokerto. Gas with a helium carrier with a flow rate of $1.37 \mathrm{~mL} / \mathrm{min}$, injector temperature of $250^{\circ} \mathrm{C}$, column temperature programmed from $100-270^{\circ} \mathrm{C}$ (Shimadzu 2008). The column used for GC programme was elite-1 (100\% Dimethylpolysiloxane), 30 x $0.25 \mathrm{~mm}$ x $1 \mu$ mdf. The equipment was GC Clarus 500 Perkin Elmer. The carrier gas used in the GC-MS program was helium 1 $\mathrm{ml} / \mathrm{minute}$ (split: 10:1). The detector used was mass detector (turbo mass gold-Perkin Elmer), the software was turbo mass 5.4.2. $2 \mu \mathrm{L}$ of the sample injected into the GCMS. The initial oven temperature was $110^{\circ} \mathrm{C}$, and it was kept hold for 2 mins, and it was raised to $200^{\circ} \mathrm{C}$ at the rate of $10^{\circ} \mathrm{C}$ per min without holding.

Further, it was raised to $280^{\circ} \mathrm{C}$ at the rate of $5^{\circ} \mathrm{C}$ per minute and kept hold for 9 minutes. The injector temperature was $250^{\circ} \mathrm{C}$. The total GC running time was 36 minutes. The library used in the MS was NIST VersionYear 2005. The inlet line temperature of the MS program was $200^{\circ} \mathrm{C}$, and the source temperature was $200^{\circ} \mathrm{C}$. The electron energy used was $70 \mathrm{eV}$; the mass scan $(\mathrm{m} / \mathrm{z})$ was 45-450. The total MS running time was 36 minutes (Mohan et al. 2015). This procedure repeated in three times. The GC-MS spectrum confirmed the presence of various components with different retention times, as illustrated in Figure 4-11. The mass spectrometer analyzes the compounds eluted at different times to identify the nature and structure of the compounds. The large compound fragments into small compounds giving rise to the appearance of peaks at different $\mathrm{m} / \mathrm{z}$ ratios. These mass spectra are a fingerprint of that compound that can identify from the data library.

\section{Antimicrobial activity}

The antimicrobial assays were conducted using agar well disc (Whatman No.1 filter paper with $9 \mathrm{~mm}$ diameter) diffusion method to obtain the values of the leaves extract of $C$. roseus cultivars against microorganisms throughout this research. Bacterial and fungal isolates (E. coli, $S$. aureus, A. flavus, and A. niger), which were obtained from the Department Microbiology, Division of Food and Nutrition Culture Collection, Gadjah Mada University, Yogyakarta, Indonesia. A concentration of $10 \mathrm{mg} / \mathrm{mL}$ prepared for all the leaves cultivar extracts by dissolving in $100 \%$ dimethyl sulfoxide (DMSO). Ten $\mathrm{mL}$ of nutrient agar (NA) and sabouraud dextrose agar (SDA) (for bacterial and fungal isolates, respectively) were poured into sterile Petri dishes $(90 \mathrm{~mm})$ and allowed to set. Put $100 \mu \mathrm{L}$ microbes, the microbes leveled with drugalsky. Leaves methanol extract dropped by $50 \mu \mathrm{L}$ for each cultivar with a concentration of $1000 \mathrm{ppm}$ on each paper disc, then disc paper it is placed on the agar medium under aseptic condition. The chloramphenicol $(10 \mu \mathrm{g} / \mathrm{mL})$ was used as a positive control for bacteria and fluconazole $(50 \mu \mathrm{g} / \mathrm{mL})$ positive control for fungi. After allowed to stand for 30 minutes, the Petri dishes incubated at $37^{\circ} \mathrm{C}$ for 24 hours. This procedure repeated in triplicate, and the mean value for inhibition zone diameters (IZDs) was measured and calculated (Davis and Stout 1971).

\section{Data analysis}

Data analysis of the research consist: (i) Analysis of interpretation mass spectrum of GC-MS conducted using the database of National Institute Standard and Techniques (NIST). The relative percentage amount of each component calculated by comparing its average peak area to the total areas. The spectrum of the unknown element compared with the spectrum of the known component inherent in the NIST library. The name, molecular weight, and structure of the details of the test materials were ascertained (Karthikeyan and Dhanapal, 2016). The relative percentage amount of each component calculated by comparing its average peak area to the total areas. The spectrum of the unknown element compared with the spectrum of the component stored in the NIST library version. It is done to determine whether these plant species contain any individual compound or group of compounds, which may substantiate its current commercial and traditional use as medicine. (ii) Analysis of inhibition microbial zone diameters (IZDs) was using analysis of variance (ANOVA), and the significant differences between the treatments were analyzed by Duncan test at a $95 \%$ confidence level.

\section{RESULTS AND DISCUSSION}

Morphological and phytochemical diversity of eight cultivars of C. roseusCatharanthus roseus plants obtained in this research classified into eight cultivars based on the 
color of the corolla flowers (Table 2). The eight cultivars of C. roseus in anatomical dan physiological characters have been reported in research before. All cultivar have different significant characters in anatomical and physiological aspects. The detection of different cultivars based on the color of the petals and eyes of the flower and the middle shade (Samiyarsih et al. 2019). The eight cultivars of C. roseus have the color of corolla (petals), which is White with white eye with yellow center, Milky white with red radiating eye with small red center, Whitish pink with pink radiating eye with yellow center, Pinkish red with pink radiating eye with yellow center, Dark pink with white large radiating base with yellow center, Pink with dark pink radiating eye with pink center, Purple blue with white large radiating base with yellow center, and Pale pink with white eye with yellow center (Figure 2). There is nothing correlation between the morfology and color of the corolla flower with phytochemical content of leaves methanol extract and abundance of major phytochemical compounds of eight C. roseus cultivars (Figure 3.).

\section{Evaluated of phytochemical component by Gas}

Chromatography-Mass Spectrum Analysis (GC-MS)

The active phytochemicals with their retention time, molecular formula, molecular weight, and concentration (\%) presented in Table 3-10 and Figure 3-11.

GC-MS spectrum is based on mass to charge ration $(\mathrm{m} / \mathrm{z})$ of the compounds. Each compound has a unique mass spectrum (molecular fingerprint) based on which compounds identified (Muthulakshmi et al. 2012; Sermakkani and Thangapandian 2012). GC-MS were promising techniques for separation, identification, and structural determination of phytochemicals. GC-MS is a powerful technique used for many applications, which is very highly sensitive and specific (Hussein et al. 2017). Generally, its form oriented towards the particular detection and potential identification of compounds based on the molecular mass in a complex mixture. The combination of a principle separation technique (GC) with the best identification technique (MS) made GC-MS an ideal analysis for volatile compounds (Karthishwaran et al. 2012).

Table 2. The classification eight cultivars of Catharanthus roseus based on the petal color and eye color of the corolla flowers

\begin{tabular}{cll}
\hline Code & $\begin{array}{l}\text { Name of } \\
\text { cultivars }\end{array}$ & Petal color eye color \\
\hline P1 & White & White-eye with yellow center \\
P2 & Milky white & Red radiating eye with a small red center \\
P3 & Whitish pink Pink radiating eye with yellow center \\
P4 & Pinkish red & Pink radiating eye with yellow center \\
U1 & Dark pink & White large radiating base with yellow center \\
U2 & Pink & Dark pink radiating eye with a pink center \\
U3 & Purple blue & White large radiating base with yellow center \\
U4 & Pale pink & White-eye with yellow center \\
\hline
\end{tabular}

Table 3. Group of major phytochemical compounds identified in the methanolic extract of Catharanthus roseus cultivars by GC-MS analysis

\begin{tabular}{|c|c|c|c|c|c|c|c|c|c|}
\hline \multirow[t]{2}{*}{ Code } & \multirow{2}{*}{$\begin{array}{c}\text { Class of chemical } \\
\text { compound }\end{array}$} & \multicolumn{8}{|c|}{ Cultivars } \\
\hline & & Dark pink & Pink & Purple blue & Pale pink & White & Milky white & Whitish pink & Pinkish red \\
\hline $\mathrm{a}$ & Essential oil & + & +++++ & + & ++++ & + & ++++ & +++++ & +++ \\
\hline $\mathrm{b}$ & Alkaloid & +++ & + & + & - & - & - & + & - \\
\hline $\mathrm{c}$ & Palmitic acid & + & - & + & - & - & - & + & + \\
\hline $\mathrm{d}$ & Linoleic acid & ++ & + & ++ & + & ++ & +++ & ++ & + \\
\hline $\mathrm{e}$ & Terpenoid & ++ & + & ++ & + & +++ & +++ & + & + \\
\hline $\mathrm{f}$ & Asam amino & + & - & - & - & - & - & - & + \\
\hline $\mathrm{g}$ & Aldehyde & + & - & - & - & - & - & - & - \\
\hline $\mathrm{h}$ & Fatty acid & + & - & - & - & +++ & + & + & - \\
\hline $\mathrm{i}$ & Nilam oil & + & - & - & - & - & - & - & - \\
\hline $\mathrm{j}$ & Aromatic compound & ++ & + & +++ & +++ & + & + & + & +++ \\
\hline $\mathrm{k}$ & Carotenoids & + & - & - & - & - & - & - & - \\
\hline 1 & Malea acid & - & + & - & - & - & - & - & - \\
\hline $\mathrm{m}$ & Mentil acetat & - & + & - & - & - & - & - & - \\
\hline $\mathrm{n}$ & Hidrocarbon & - & + & - & - & - & + & - & - \\
\hline o & Acetic acid & - & - & + & - & + & & - & + \\
\hline $\mathrm{p}$ & Aldehydes/alcohol & - & - & - & - & + & + & + & + \\
\hline$q$ & Myristoleic acid & - & - & - & - & - & + & - & + \\
\hline $\mathrm{r}$ & Phenolic & + & + & + & + & + & + & + & + \\
\hline & Total & $12(66 \%)$ & $9(50 \%)$ & $8(44 \%)$ & $5(27 \%)$ & $8(44 \%)$ & $9(50 \%)$ & $9(50 \%)$ & $10(55 \%)$ \\
\hline
\end{tabular}

Note: The abundance of phytochemical classes in every cultivar of Catharanthus roseus. (-): absence of compound; (+), (++): medium, and $(+++)$ or above is high 

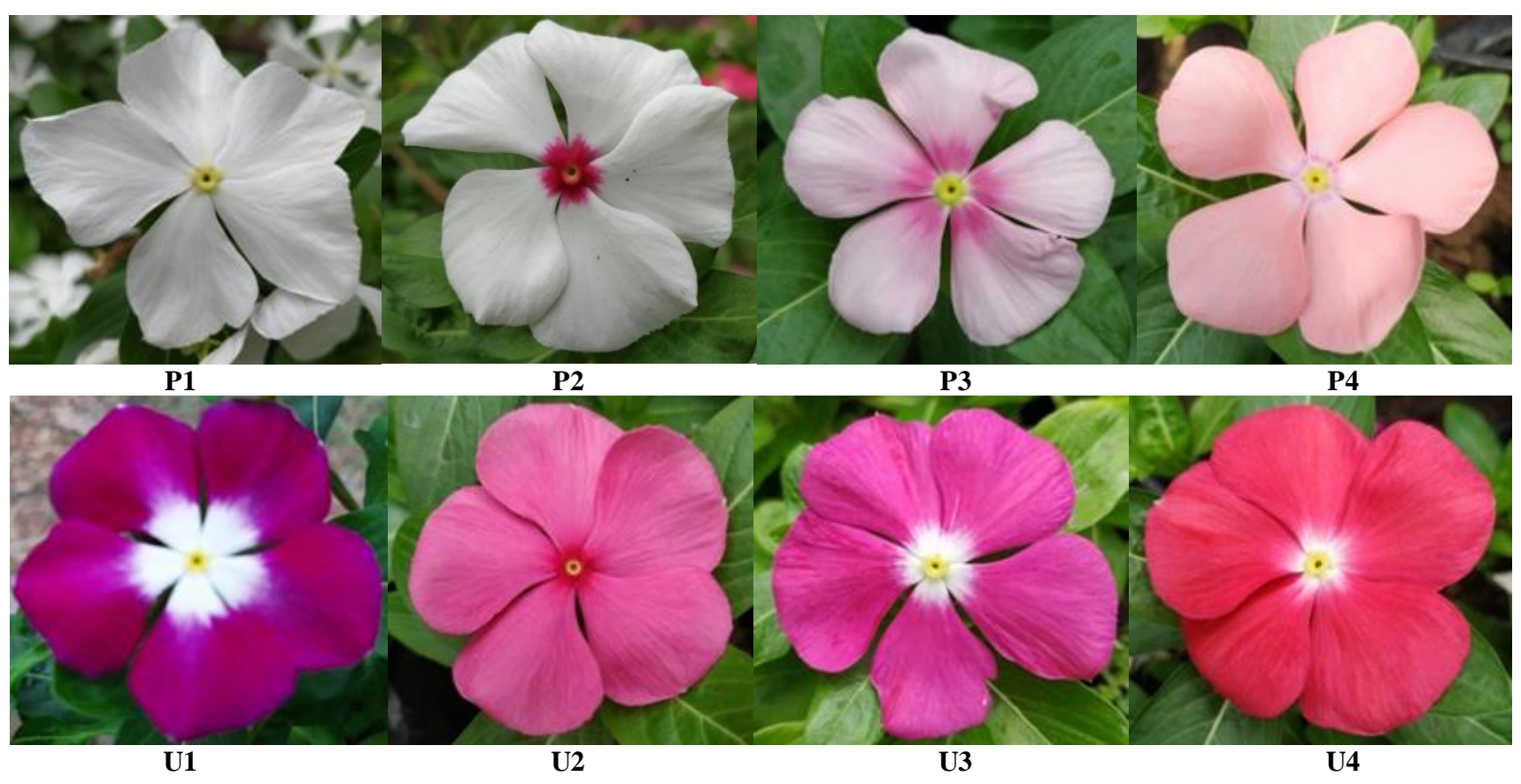

Figure 2. Photographs of the color flowers the eight cultivars of Catharanthus roseus. Note: Cultivars of P1-P4 and U1-U4 were distinguished based on the color of the corolla, as indicated in Table 2.

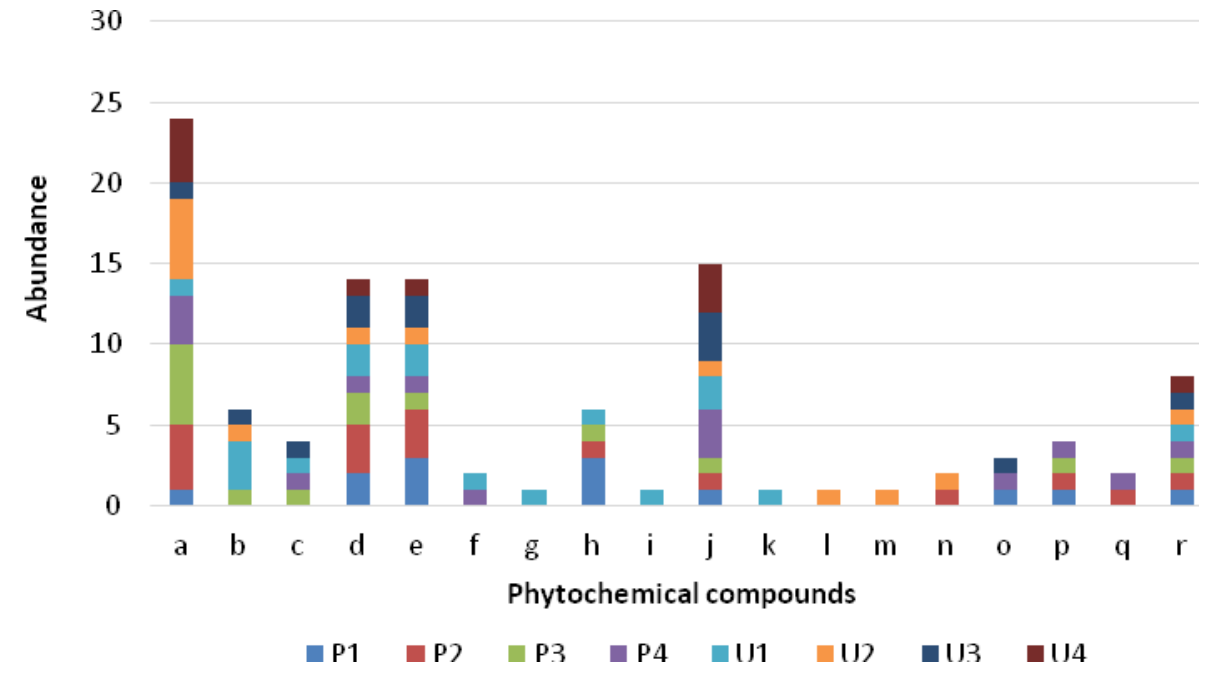

Figure 3. An abundance of major phytochemical compounds of eight Catharanthus roseus cultivars. Note. a-r: a primary phytochemical compound in Table 3.; P1-U4: the identity of each cultivar in Table 2

This method can be used to identify a compound, either one component or mixture. Mass spectrophotometers used in determining fragmentation and molecules and identifying components contained in small quantities. Retention time (RT) is the time needed for a compound to move through the column to the detector. Retention time measurement based on the time when the sample injected until the sample showed a maximum peak height. GC-MS is a combined technique used to identify different substances within the sample. It works on the separation of the individual compound by GC according to their RT, and the separated compounds further analyzed at a molecular level by MS (Painuli et al. 2016). Pinho et al. (2009) reported the results of research on the constituents of $C$. roseus volatiles using HS-SPME fiber combined with gas chromatography-mass spectrometry (GC-MS) analysis. It incorporates extraction, concentration, and sample introduction into a single step. Using the DVB/PDMS fibre 
73 compounds were identified for the first time in fresh flowers, stems, and leaves of $C$. roseus. Some of these compounds have an important bioactivity role in the human body. Phytochemical profiles from the analysis with GCMS spectrum of eight $C$. roseus cultivars presented in Figure 4-9.

The zone of inhibition of eight of $C$. roseus cultivars observed in leaf methanolic extract against the microbes $E$. coli, S. aureus, A. flavus, and A. niger. Antimicrobial assay of the leaves extracts of $C$. roseus cultivars (Table 12,
Figure 12) reveals that at $10 \mathrm{mg} / \mathrm{mL}$, the extracts showed activity against the bacterial and fungal test isolates with inhibition zones ranging from $6.40-22.00 \mathrm{~mm}$ and 3.35 $8.20 \mathrm{~mm}$ respectively. All of the antimicrobial properties are significantly different at the Duncan test $(\mathrm{p}<0.05)$. The extracts of the leaves from $C$. roseus represent a dependable source of phytochemicals and bioactive compounds, evidenced by the wide range of compounds with diverse biological properties present in these extracts.

Table 4. Phytocomponents identified in the methanolic extract of the leaf of Catharanthus roseus cult. "Dark Pink" by GC-MS analysis

\begin{tabular}{|c|c|c|c|c|c|c|}
\hline $\begin{array}{l}\text { Retention } \\
\text { time }\end{array}$ & Area & $\begin{array}{c}\text { Area } \\
\%\end{array}$ & Name & $\begin{array}{l}\text { Molecular } \\
\text { formula }\end{array}$ & $\begin{array}{c}\text { Molecular } \\
\text { weight }\left(\text { g.mol }^{-1}\right)\end{array}$ & Group \\
\hline 8.13 & 61257 & 1.19 & Beta tumeron & $\mathrm{C}_{15} \mathrm{H}_{22} \mathrm{O}$ & 218.33 & Essential oil \\
\hline 10.31 & 118637 & 2.68 & Coronaridine & $\mathrm{C}_{21} \mathrm{H}_{26} \mathrm{~N}_{2} \mathrm{O}_{2}$ & 338.40 & Alkaloid \\
\hline 9.72 & 271255 & 5.27 & Hexadecanoic acid, methyl ester (CAS) & $\mathrm{C}_{16} \mathrm{H}_{32} \mathrm{O}_{2}$ & 270.50 & Palmitic acid \\
\hline 10.76 & 314029 & 6.10 & $9,12,15$-Octadecatrienoic acid, methyl ester (CAS) & $\mathrm{C}_{19} \mathrm{H}_{32} \mathrm{O}_{2}$ & 292.50 & Linoleic acid \\
\hline 10.82 & 3761139 & 73.00 & 3,7,11,15-Tetramethyl-2-hexadecane-1-ol & $\mathrm{C}_{20} \mathrm{H}_{40} \mathrm{O}$ & 296.50 & Terpenoid alcohol \\
\hline 7.80 & 51732 & 1.08 & L-Alanine, N-acetyl- (CAS) & $\mathrm{C}_{3} \mathrm{H}_{7} \mathrm{NO}_{2}$ & 131.13 & Asam amino \\
\hline 8.27 & 68932 & 1.43 & Beta Ionol & $\mathrm{C}_{13} \mathrm{H}_{22} \mathrm{O}$ & 194.31 & Aldehyde \\
\hline 10.82 & 3926229 & 71.16 & 2-Hexadecen-1-ol, 3,7,11,15-tetramethyl & $\mathrm{C}_{20} \mathrm{H}_{40} \mathrm{O}$ & 296.50 & Aromatic compounds \\
\hline 10.98 & 197087 & 3.03 & 8,11,14-Eicosatrienoic acid, (Z,Z,Z)- (CAS) & $\mathrm{C}_{20} \mathrm{H}_{34} \mathrm{O}_{2}$ & 302.65 & Fatty acid \\
\hline 11.02 & 215904 & 3.32 & Neophytadiene & $\mathrm{C}_{20} \mathrm{H}_{38}$ & 278.5 & Terpenoid \\
\hline 11.08 & 46466 & 0.71 & Patchouli alcohol & $\mathrm{C}_{15} \mathrm{H}_{26} \mathrm{O}$ & 222.36 & Aromatic compounds \\
\hline 11.39 & 54737 & 0.84 & Lycopene 7 & $\mathrm{C}_{40} \mathrm{H}_{56}$ & 536.87 & $\begin{array}{l}\text { Carotenoid } \\
\text { hydrocarbon }\end{array}$ \\
\hline
\end{tabular}

Table 6. Phytocomponents identified in the methanolic extract of the leaf of Catharanthus roseus cult. "Purple Blue" by GC-MS analysis

\begin{tabular}{|c|c|c|c|c|c|c|}
\hline $\begin{array}{l}\text { Retention } \\
\text { time }\end{array}$ & Area & $\begin{array}{c}\text { Area } \\
\%\end{array}$ & Name & $\begin{array}{c}\text { Molecular } \\
\text { formula }\end{array}$ & $\begin{array}{c}\text { Molecular } \\
\text { weight }\left(\text { g.mol }{ }^{-1}\right)\end{array}$ & Group \\
\hline 9.71 & 226583 & 6.34 & Hexadecanoic acid, methyl ester (CAS) & $\mathrm{C}_{16} \mathrm{H}_{32} \mathrm{O}_{2}$ & 270.50 & Palmitic acid \\
\hline 9.92 & 36731 & 1.03 & 9-Octadecenoic acid (Z)- (CAS) & $\mathrm{C}_{18} \mathrm{H}_{34} \mathrm{O}_{2}$ & 282.50 & Linoleic acid \\
\hline 10.75 & 254199 & 7.12 & 9,12,15-Octadecatrienoic acid, methyl ester (CAS) & $\mathrm{C}_{19} \mathrm{H}_{32} \mathrm{O}_{2}$ & 292.50 & Linoleic acid \\
\hline 10.82 & 2353584 & 65.90 & $\begin{array}{l}\text { 2-Hexadecen-1-OL, 3,7,11,15-Tetramethyl-, [R- } \\
{\left[\mathrm{R}^{*}, \mathrm{R}^{*} \text { - (E)]]- (T-Phytol) }\right.}\end{array}$ & $\mathrm{C}_{20} \mathrm{H}_{40} \mathrm{O}$ & 296.50 & Terpenoid alcohol \\
\hline 3.95 & 51772 & 1.31 & Piperidine, 3-phenyl- & $\mathrm{C}_{11} \mathrm{H}_{15} \mathrm{~N}$ & 161.24 & Aromatic compounds \\
\hline 8.13 & 51432 & 1.30 & Beta tumeron & $\mathrm{C}_{15} \mathrm{H}_{22} \mathrm{O}$ & 218.33 & Essential oil \\
\hline 11.12 & 75697 & 1.92 & 1,2-dihydro-8-hydroxylinalool & $\mathrm{C}_{10} \mathrm{H}_{20} \mathrm{O}_{2}$ & 172.26 & Aromatic compounds \\
\hline 11.02 & 179902 & 5.22 & 3,7,11,15-Tetramethyl-2-hexadecane-1 & $\mathrm{C}_{20} \mathrm{H}_{40} \mathrm{O}$ & 296.50 & Terpenoid alcohol \\
\hline 11.15 & 84766 & 2.46 & D-Galactitol-5-O-hexyl- & $\mathrm{C}_{12} \mathrm{H}_{26} \mathrm{O}_{6}$ & 266.33 & Aromatic compounds \\
\hline 12.11 & 38705 & 1.12 & Vitamin E acetate & $\mathrm{C}_{31} \mathrm{H}_{52} \mathrm{O}_{3}$ & 472.70 & Acetic acid \\
\hline
\end{tabular}

Table 7. Phytocomponents identified in the methanolic extract of the leaf of Catharanthus roseus cult. "Pale Pink" by GC-MS analysis

\begin{tabular}{|c|c|c|c|c|c|c|}
\hline $\begin{array}{l}\text { Retention } \\
\text { time }\end{array}$ & Area & $\underset{\%}{\text { Area }}$ & Name & $\begin{array}{l}\text { Molecular } \\
\text { formula }\end{array}$ & $\begin{array}{c}\text { Molecular } \\
\text { weight }(\text { g.mol } \\
\text { wit) }\end{array}$ & Group \\
\hline 8.13 & 43250 & 0.92 & Beta tumeron & $\mathrm{C}_{15} \mathrm{H}_{22} \mathrm{O}$ & 218.33 & Essential oil \\
\hline 8.17 & 13862 & 0.29 & Ar turmerone & $\mathrm{C}_{15} \mathrm{H}_{20} \mathrm{O}$ & 216.32 & Essential oil \\
\hline 10.83 & 3303330 & 70.26 & $\begin{array}{l}\text { 2-Hexadecen-1-ol, 3,7,11,15-tetramethyl-, [R- } \\
{\left[\mathrm{R}^{*}, \mathrm{R}^{*} \text { - (E)]]- (CAS) }\right.}\end{array}$ & $\mathrm{C}_{20} \mathrm{H}_{40} \mathrm{O}$ & 296.50 & Aromatic compounds \\
\hline 11.13 & 75104 & 1.60 & Ethyl linoleate & $\mathrm{C}_{20} \mathrm{H}_{36}$ & 308.00 & Linoleic acid \\
\hline 12.13 & 27189 & 0.58 & .alpha.-Tocopherol-.beta.-D-mannose & $\mathrm{C}_{35} \mathrm{H}_{60} \mathrm{O}_{7}$ & 592.80 & Essential oil \\
\hline 8.41 & 50579 & 1.10 & Alpha tumeron & $\mathrm{C}_{15} \mathrm{H}_{22} \mathrm{O}$ & 218.33 & Essential oil \\
\hline 11.35 & 20843 & 0.45 & Neophytadiene & $\mathrm{C}_{20} \mathrm{H}_{38}$ & 278.50 & Terpenoid \\
\hline 11.13 & 30949 & 0.74 & Bicyclo[3.3.1]non-6-ene-3,9-dione & $\mathrm{C}_{9} \mathrm{H}_{10} \mathrm{O}_{2}$ & 150.17 & Aromatic compounds \\
\hline 12.53 & 39905 & 0.95 & Methyl trans-community & $\mathrm{C}_{21} \mathrm{H}_{32} \mathrm{O}_{2}$ & 316.50 & Aromatic compounds \\
\hline
\end{tabular}


Table 8. Phytocomponents identified in the methanolic extract of the leaf of Catharanthus roseus cult. "White" by GC-MS analysis

\begin{tabular}{|c|c|c|c|c|c|c|}
\hline $\begin{array}{c}\text { Retention } \\
\text { time }\end{array}$ & Area & $\begin{array}{c}\text { Area } \\
\%\end{array}$ & Name & $\begin{array}{l}\text { Molecular } \\
\text { formula }\end{array}$ & $\begin{array}{c}\text { Molecular } \\
\text { weight }\left(\mathrm{g} \cdot \mathrm{mol}^{-1}\right)\end{array}$ & Group \\
\hline 10.98 & 597590 & 11.01 & 9,12,15-Octadecatrien-1-ol (CAS) & $\mathrm{C}_{19} \mathrm{H}_{32} \mathrm{O}_{2}$ & 292.50 & Linoleic acid \\
\hline 11.04 & 453852 & 8.36 & 3,7,11,15-Tetramethyl-2-hexadecane-1-ol & $\mathrm{C}_{20} \mathrm{H}_{40} \mathrm{O}$ & 296.50 & Terpenoid alcohol \\
\hline 11.13 & 167109 & 3.08 & 6,9,12-Octadecatrienoic acid, methyl ester (CAS) & $\mathrm{C}_{19} \mathrm{H}_{32} \mathrm{O}_{2}$ & 292.50 & Linoleic acid \\
\hline 11.29 & 90367 & 1.67 & Octanoic acid, phenylmethyl ester (CAS) & $\mathrm{C}_{18} \mathrm{H}_{34} \mathrm{O}_{2}$ & 282.50 & Fatty acid \\
\hline 11.37 & 36281 & 0.67 & (Z)6-Pentadecen-1-ol & $\mathrm{C}_{15} \mathrm{H}_{30} \mathrm{O}$ & 226.40 & Fatty acid \\
\hline 12.08 & 49082 & 0.90 & Vitamin $\mathrm{E}$ acetate & $\mathrm{C}_{31} \mathrm{H}_{52} \mathrm{O}_{3}$ & 472.70 & Acetic acid \\
\hline 8.13 & 73782 & 0.93 & Beta tumeron & $\mathrm{C}_{15} \mathrm{H}_{22} \mathrm{O}$ & 218.33 & Essential oil \\
\hline 8.18 & 44430 & 0.56 & Ar turmerone & $\mathrm{C}_{15} \mathrm{H}_{20} \mathrm{O}$ & 216.32 & Essential oil \\
\hline 8.29 & 35031 & 0.44 & .beta.-Ionol & $\mathrm{C}_{13} \mathrm{H}_{22} \mathrm{O}$ & 194.31 & Aldehyde \\
\hline 8.42 & 45819 & 0.58 & Alpha tumeron & $\mathrm{C}_{15} \mathrm{H}_{22} \mathrm{O}$ & 218.33 & Essential oil \\
\hline 9.93 & 60076 & 0.76 & Docosanoic acid (CAS) & $\mathrm{C}_{22} \mathrm{H}_{44} \mathrm{O}_{2}$ & 340.60 & Fatty acid \\
\hline 11.03 & 375906 & 4.73 & Neophytadiene & $\mathrm{C}_{20} \mathrm{H}_{38}$ & 278.50 & Terpenoid \\
\hline 10.82 & 4052663 & 63.26 & 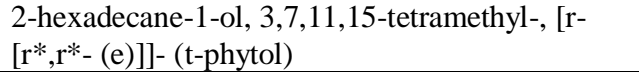 & $\mathrm{C}_{20} \mathrm{H}_{40} \mathrm{O}$ & 296.50 & Terpenoid alcohol \\
\hline
\end{tabular}

Table 9. Phytocomponents identified in the methanolic extract of the leaf of Catharanthus roseus cult. "Milky White" by GC-MS analysis

\begin{tabular}{|c|c|c|c|c|c|c|}
\hline $\begin{array}{l}\text { Retention } \\
\text { time }\end{array}$ & Area & $\begin{array}{c}\text { Area } \\
\%\end{array}$ & Name & $\begin{array}{l}\text { Molecular } \\
\text { formula }\end{array}$ & $\begin{array}{c}\text { Molecular } \\
\text { weight }(\text { g.mol } \\
-1 \text { ) }\end{array}$ & Group \\
\hline 4.27 & 41052 & 0.66 & Camphor (CAS) & $\mathrm{C}_{10} \mathrm{H}_{16} \mathrm{O}$ & 152.23 & Terpenoid \\
\hline 8.13 & 38229 & 0.62 & Beta tumeron & $\mathrm{C}_{15} \mathrm{H}_{22} \mathrm{O}$ & 218.33 & Essential oil \\
\hline 10.76 & 471857 & 7.63 & $\begin{array}{l}\text { 9,12,15-Octadecatrienoic acid, methyl ester, } \\
\text { (Z,Z,Z)- (CAS) }\end{array}$ & $\mathrm{C}_{19} \mathrm{H}_{32} \mathrm{O}_{2}$ & 292.50 & Linoleic acid \\
\hline 10.83 & 3393626 & 54.90 & $\begin{array}{l}\text { 2-Hexadecen-1-OL,3,7,11,15-Tetramethyl-, [R- } \\
{\left[\mathrm{R}^{*}, \mathrm{R}^{*} \text { - (E)]]- (T-Phytol) }\right.}\end{array}$ & $\mathrm{C}_{20} \mathrm{H}_{40} \mathrm{O}$ & 296.50 & Diterpene alcohol \\
\hline 10.98 & 795981 & 12.88 & $\begin{array}{l}\text { 9,12,15-Octadecatrienoic acid, methyl ester, } \\
\text { (Z,Z,Z)- (CAS) }\end{array}$ & $\mathrm{C}_{20} \mathrm{H}_{40} \mathrm{O}$ & 296.50 & Linoleic acid \\
\hline 11.04 & 412811 & 6.68 & Neophytadiene & $\mathrm{C}_{20} \mathrm{H}_{38}$ & 278.50 & Hydrocarbon Alkana \\
\hline 11.35 & 21084 & 0.34 & Cic-D-Dihydrocarveol & $\mathrm{C}_{10} \mathrm{H}_{18} \mathrm{O}$ & 154.25 & Terpenoid \\
\hline 11.40 & 28398 & 0.46 & Farnesol & $\mathrm{C}_{15} \mathrm{H}_{26} \mathrm{O}$ & 222.37 & Isopropanol alcohol \\
\hline 12.09 & 28927 & 0.47 & Alpha.-Tocopherol-.beta.-D-mannose & $\mathrm{C}_{35} \mathrm{H}_{60} \mathrm{O}_{7}$ & 592.80 & Essential oil \\
\hline 8.18 & 29515 & 0.69 & Ar-Tumerone & $\mathrm{C}_{15} \mathrm{H}_{20} \mathrm{O}$ & 216.32 & Essential oil \\
\hline 8.42 & 22125 & 0.52 & Alpha.-turmerone & $\mathrm{C}_{15} \mathrm{H}_{22} \mathrm{O}$ & 218.33 & Essential oil \\
\hline 10.73 & 44190 & 1.09 & Ethyl linoleate & $\mathrm{C}_{20} \mathrm{H}_{36}$ & 308.00 & Linoleic acid \\
\hline 11.03 & 210032 & 5.18 & citronellyl 2-methyl propanoate & $\mathrm{C}_{14} \mathrm{H}_{26} \mathrm{O}_{2}$ & 226.35 & Myristoleic acid \\
\hline 11.15 & 33372 & 0.82 & Guanosine (CAS) & $\mathrm{C}_{10} \mathrm{H}_{13} \mathrm{~N}_{5} \mathrm{O}_{5}$ & 283.24 & Nuclear acid \\
\hline 11.81 & 31801 & 0.78 & Dodecanoic acid, 10 -undecen-1-yl ester & $\mathrm{C}_{23} \mathrm{H}_{44} \mathrm{O}_{2}$ & 352.60 & Fatty acid \\
\hline
\end{tabular}

Table 10. Phytocomponents identified in the methanolic extract of the leaf of Catharanthus roseus cult. "Withish Pink" by GC-MS analysis

\begin{tabular}{|c|c|c|c|c|c|c|}
\hline $\begin{array}{l}\text { Retention } \\
\text { time }\end{array}$ & Area & $\begin{array}{c}\text { Area } \\
\%\end{array}$ & Name & $\begin{array}{c}\text { Molecular } \\
\text { formula }\end{array}$ & $\begin{array}{c}\text { Molecular } \\
\text { weight }\left(\mathrm{g} \cdot \mathrm{mol}^{-1}\right)\end{array}$ & Group \\
\hline 8.13 & 90248 & 1,04 & Beta Tumerone & $\mathrm{C}_{15} \mathrm{H}_{22} \mathrm{O}$ & 218.33 & Essential oil \\
\hline 8.17 & 40275 & 0,46 & Ar-Tumerone & $\mathrm{C}_{15} \mathrm{H}_{20} \mathrm{O}$ & 216.32 & Essential oil \\
\hline 8.41 & 28920 & 0,33 & Alpha.-turmerone & $\mathrm{C}_{15} \mathrm{H}_{22} \mathrm{O}$ & 218.33 & Essential oil \\
\hline 9.72 & 431666 & 4,95 & Hexadecanoic acid, methyl ester (CAS) & $\mathrm{C}_{16} \mathrm{H}_{32} \mathrm{O}_{2}$ & 270.50 & Palmitic acid \\
\hline 10.83 & 4887676 & 56,07 & $\begin{array}{l}\text { 2-Hexadecen-1-OL, 3,7,11,15-Tetramethyl-, [R- } \\
{\left[\mathrm{R}^{*}, \mathrm{R}^{*} \text { - (E)]]- (T-Phytol) }\right.}\end{array}$ & $\mathrm{C}_{20} \mathrm{H}_{40} \mathrm{O}$ & 296.50 & $\begin{array}{l}\text { Acyclic diterpene } \\
\text { alcohol }\end{array}$ \\
\hline 10.98 & 1878424 & 21,55 & $\begin{array}{l}\text { 9,12,15-Octadecatrienoic acid, methyl ester, } \\
\text { (Z,Z,Z)- (CAS) }\end{array}$ & $\mathrm{C}_{20} \mathrm{H}_{40} \mathrm{O}$ & 296.50 & Linoleic acid \\
\hline 7.65 & 65047 & 1,47 & 4-Pyridinecarboxamide (CAS) & $\mathrm{C}_{26} \mathrm{H}_{31} \mathrm{C}_{1} \mathrm{~N}_{6} \mathrm{O}_{2}$ & 495.00 & Essential oil \\
\hline 10.09 & 82448 & 1,86 & Piperidine, 1-nitro- (CAS) & $\mathrm{C}_{13} \mathrm{H}_{11} \mathrm{~N}_{3} \mathrm{O}_{5}$ & 289.24 & Essential oil \\
\hline 10.31 & 118637 & 2,68 & Coronaridine & $\mathrm{C}_{21} \mathrm{H}_{26} \mathrm{~N}_{2} \mathrm{O}_{2}$ & 338.40 & Alkaloid \\
\hline 11.17 & 61656 & 1,39 & L-Glutamine,N2 [ (phenyl methoxy)carbonyl]- & $\mathrm{C}_{13} \mathrm{H}_{16} \mathrm{~N}_{2} \mathrm{O}_{5}$ & 280.28 & Fatty acid \\
\hline 11.35 & 67937 & 1,54 & Citronellyl Acetate & $\mathrm{C}_{12} \mathrm{H}_{22} \mathrm{O}_{2}$ & 198.30 & Linoleic acid \\
\hline 11.03 & 403171 & 6,72 & Neophytadiene & $\mathrm{C}_{20} \mathrm{H}_{38}$ & 278.50 & Terpenoid \\
\hline
\end{tabular}


Table 11. Phytocomponents identified in the methanolic extract of the leaf of Catharanthus roseus cult. "Pinkish Red" by GC-MS analysis

\begin{tabular}{lllllll}
\hline $\begin{array}{c}\text { Retention } \\
\text { time }\end{array}$ & \multicolumn{1}{c}{ Area } & $\begin{array}{c}\text { Area } \\
\text { \% }\end{array}$ & \multicolumn{1}{c}{ Name } & $\begin{array}{c}\text { Molecular } \\
\text { formula }\end{array}$ & $\begin{array}{c}\text { Molecular } \\
\text { weight (g.mol }{ }^{-1} \text { ) }\end{array}$ & \multicolumn{1}{c}{ Group } \\
\hline 8.13 & 50841 & 0.83 & Beta Tumerone & $\mathrm{C}_{15} \mathrm{H}_{22} \mathrm{O}$ & 218.33 & Essential oil \\
10.00 & 39949 & 0.65 & O,N-Permethylated N-Acetyllysine & $\mathrm{C}_{8} \mathrm{H}_{16} \mathrm{~N}_{2} \mathrm{O}_{3}$ & 188.22 & Asam amino \\
10.06 & 48562 & 0.80 & Trimethylsilyl Derivative Of 2-Monoolein & $\mathrm{C}_{27} \mathrm{H}_{56} \mathrm{O}_{4} \mathrm{Si}_{2}$ & 500.90 & Aromatic compounds \\
10.97 & 1673520 & 27.44 & $9,12,15-O c t a d e c a t r i e n-1-o l ~(C A S)$ & $\mathrm{C}_{20} \mathrm{H}_{40} \mathrm{O}$ & 296.50 & Linoleic acid \\
11.35 & 43520 & 0.71 & Citronellyl Valerate & $\mathrm{C}_{15} \mathrm{H}_{28} \mathrm{O}_{2}$ & 240.38 & Myristoleic acid \\
11.43 & 43360 & 0.71 & Thiosulfuric acid (H2S2O3), S- (2-aminoethyl) ester & $\mathrm{C}_{2} \mathrm{H}_{6} \mathrm{NNaO}_{3} \mathrm{~S}_{2}$ & 179.20 & Acid sulfate \\
12.13 & 59965 & 0.98 & alpha-Tocopherol-.beta.-D-mannose & $\mathrm{C}_{35} \mathrm{H}_{60} \mathrm{O}_{7}$ & 592.80 & Essential oil \\
11.03 & 460974 & 8.14 & Neophytadiene & $\mathrm{C}_{20} \mathrm{H}_{38}$ & 278.50 & Aromatic compounds \\
11.13 & 48510 & 0.86 & CIS-7-Methylbicyclo[4.3.0]NON-3-ENE & $\mathrm{C}_{13} \mathrm{H}_{20} \mathrm{O}_{3}$ & 224.29 & Acetaldehyde \\
12.12 & 29716 & 0.52 & Vitamin E acetate & $\mathrm{C}_{31} \mathrm{H}_{52} \mathrm{O}_{3}$ & 472.70 & Acetic acid \\
8.17 & 25250 & 0.40 & Ar-Tumerone & $\mathrm{C}_{15} \mathrm{H}_{20} \mathrm{O}$ & 216.32 & Aromatic compounds \\
8.41 & 26906 & 0.42 & Alpha-turmerone & $\mathrm{C}_{15} \mathrm{H}_{22} \mathrm{O}$ & 218.33 & Essential oil \\
10.82 & 3492924 & 54.79 & 2-Hexadecen-1-ol, 3,7,11,15-tetramethyl CAS) & $\mathrm{C}_{20} \mathrm{H}_{40} \mathrm{O}$ & 296.50 & Palmitic acid \\
\hline
\end{tabular}

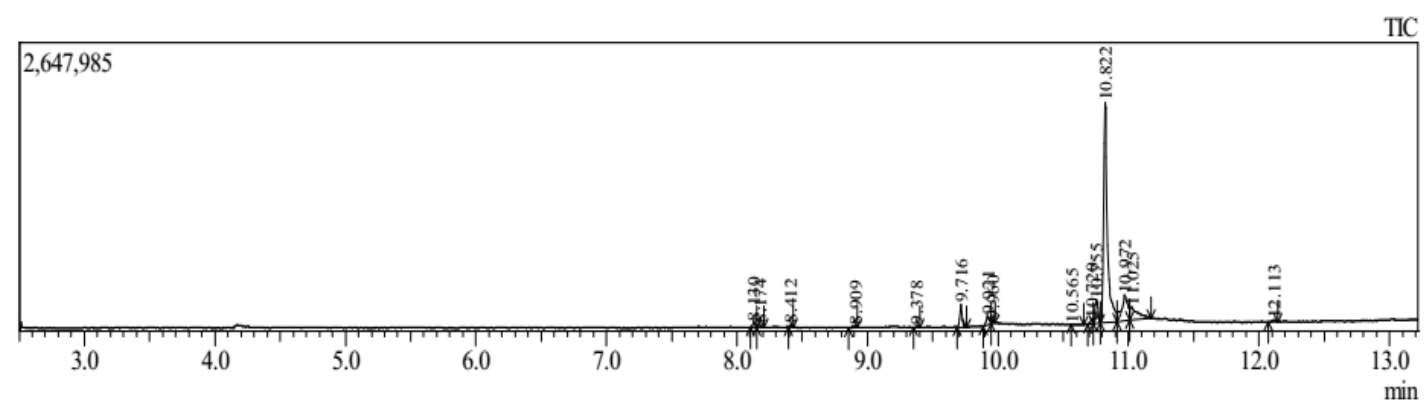

Figure 4. GC-MS spectrum of methanol extract of Catharanthus roseus cult."White"

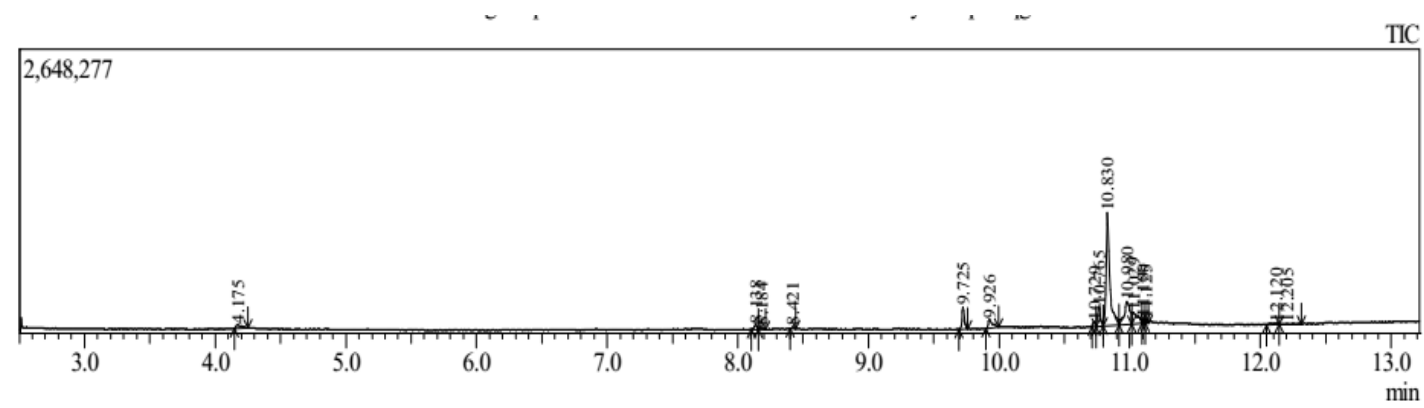

Figure 5. GC-MS spectrum of methanol extract of Catharanthus roseus cult."Milky White"

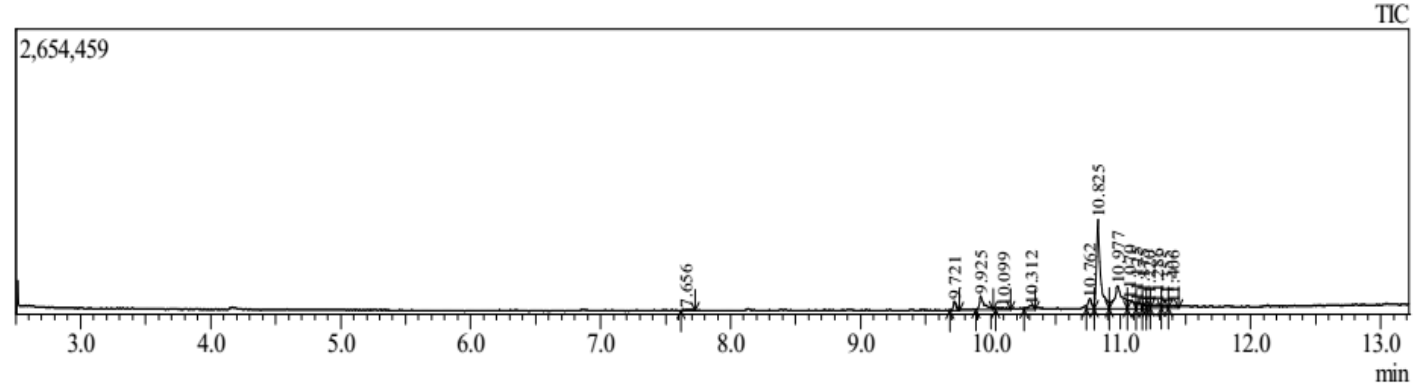

Figure 6. GC-MS spectrum of methanol extract of Catharanthus roseus cult. "Whitish Pink" 


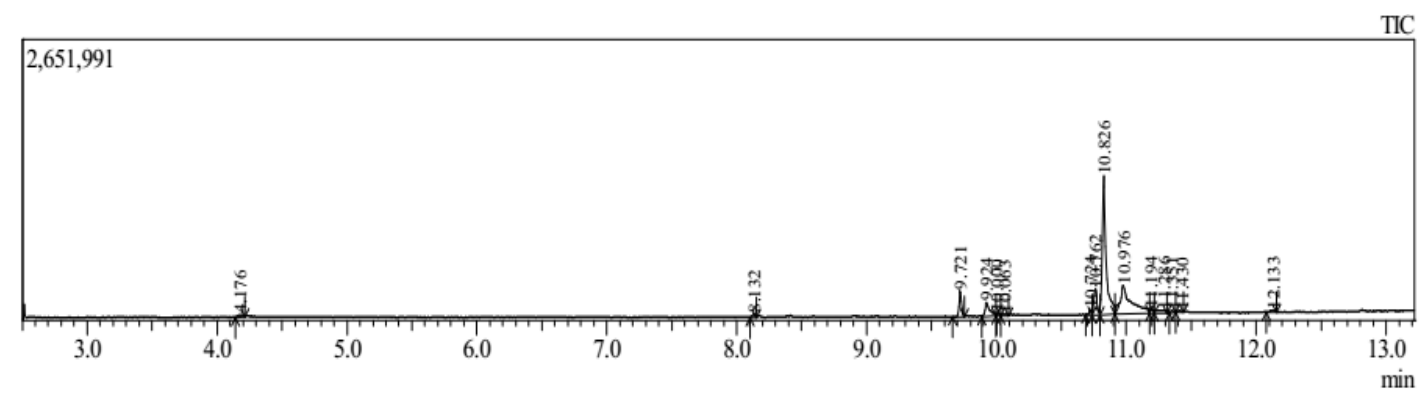

Figure 7. GC-MS spectrum of methanol extract of Catharanthus roseus cult. "Pinkish Red"

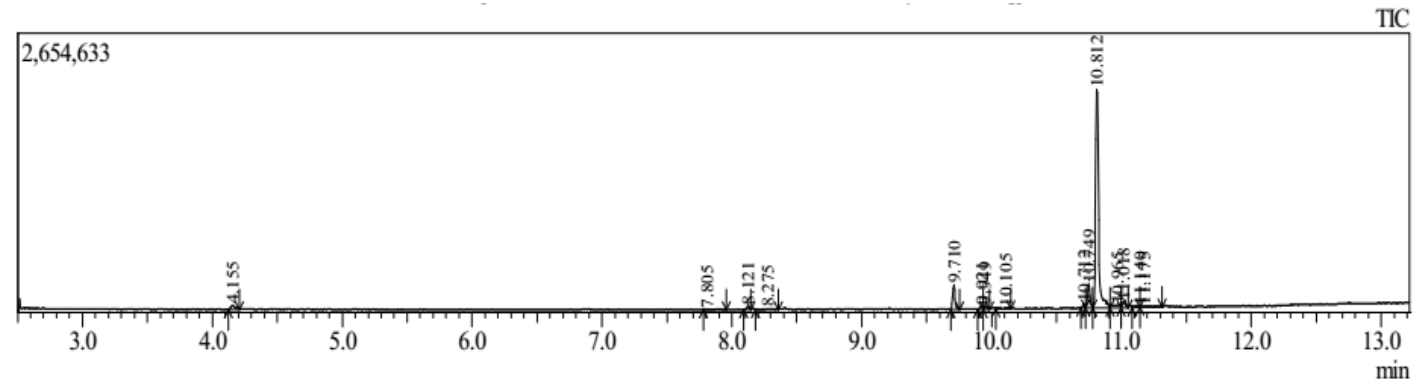

Figure. 8. GC-MS spectrum of methanol extract of Catharanthus roseus cult. "Dark Pink"

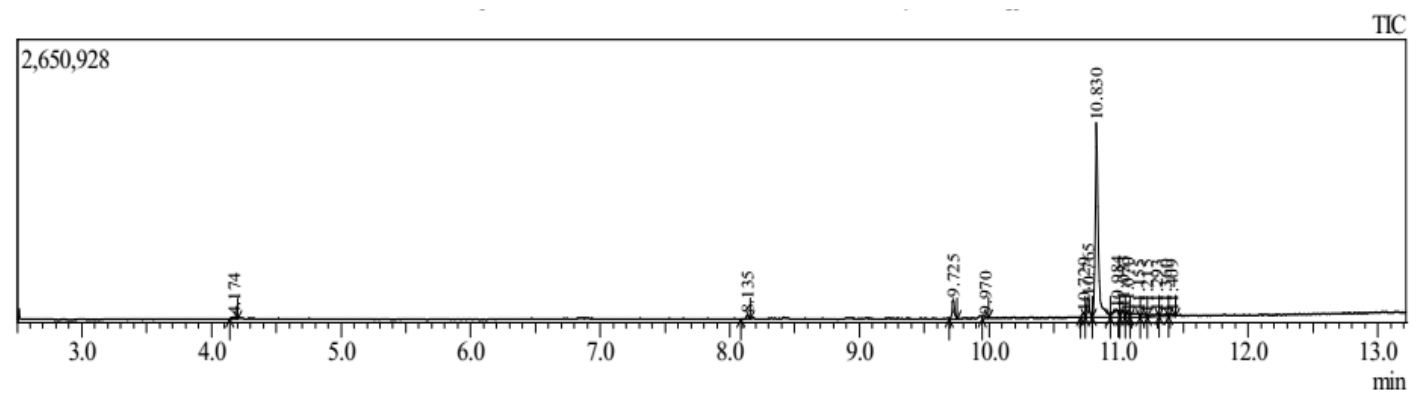

Figure. 9. GC-MS spectrum of methanol extract of Catharanthus roseus cult."Pink"

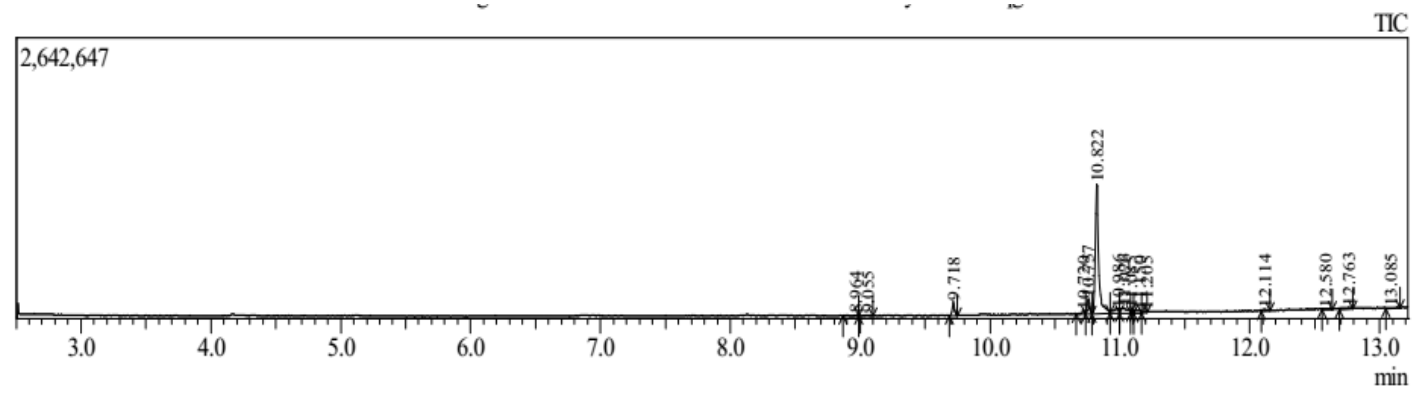

Figure. 10. GC-MS spectrum of methanol extract of Catharanthus roseus cult. "Purple Blue"

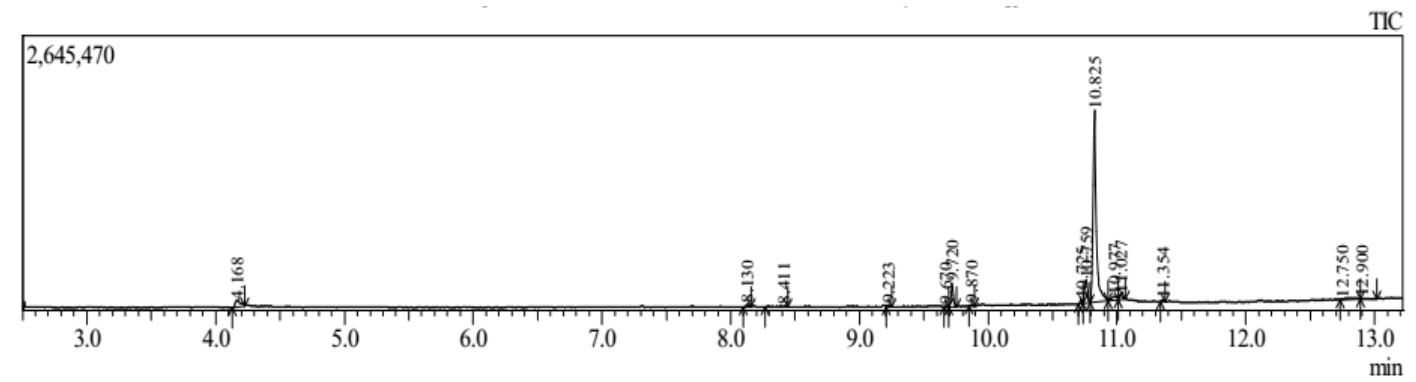

Figure. 11. GC-MS spectrum of methanol extract of Catharanthus roseus cult."Pale Pink" 
Table 12. Inhibition zone diameters (IZDs) of eight Catharanthus roseus leaves cultivars against microbes

\begin{tabular}{lcccc}
\hline \multirow{2}{*}{ Cultivars } & \multicolumn{3}{c}{ Test microbial and Inhibition zone diameters (mm \pm S.D) } \\
\cline { 2 - 5 } & $\boldsymbol{E . ~ c o l i}$ & S. aureus & A. flavus & A. niger \\
\hline White & $7.45 \pm 0.05 \mathrm{bc}$ & $14.70 \pm 0.10 \mathrm{~b}$ & $3.35 \pm 0.05 \mathrm{bc}$ & $5.55 \pm 0.15 \mathrm{bc}$ \\
Milky White & $8.25 \pm 0.86 \mathrm{bc}$ & $12.10 \pm 0.20 \mathrm{bc}$ & $4.10 \pm 0.05 \mathrm{~b}$ & $4.00 \pm 0.15 \mathrm{bc}$ \\
Whitish Pink & $6.40 \pm 0.10 \mathrm{bc}$ & $13.80 \pm 0.05 \mathrm{~b}$ & $4.20 \pm 0.13 \mathrm{~b}$ & $4.25 \pm 0.15 \mathrm{bc}$ \\
Pinkish red & $12.80 \pm 0.17 \mathrm{ab}$ & $11.30 \pm 0.17 \mathrm{c}$ & $4.80 \pm 0.13 \mathrm{~b}$ & $6.70 \pm 0.10 \mathrm{~b}$ \\
Dark Pink & $16.10 \pm 0.26 \mathrm{a}$ & $22.00 \pm 0.95 \mathrm{a}$ & $6.05 \pm 0.05 \mathrm{a}$ & $8.20 \pm 0.17 \mathrm{a}$ \\
Pink & $14.10 \pm 0.17 \mathrm{ab}$ & $18.50 \pm 0.50 \mathrm{ab}$ & $5.00 \pm 0.05 \mathrm{ab}$ & $7.60 \pm 0.20 \mathrm{ab}$ \\
Purple Blue & $11.85 \pm 0.05 \mathrm{~b}$ & $15.20 \pm 0.26 \mathrm{~b}$ & $4.60 \pm 0.05 \mathrm{~b}$ & $7.00 \pm 0.20 \mathrm{~b}$ \\
Pale Pink & $10.25 \pm 0.05 \mathrm{~b}$ & $13.60 \pm 0.15 \mathrm{~b}$ & $5.20 \pm 0.08 \mathrm{ab}$ & $6.90 \pm 0.10 \mathrm{~b}$ \\
Chloramphenicol & 23.00 & 31.00 & - & - \\
Fluconazole & - & - & 12.00 & 15.00 \\
\hline
\end{tabular}

Notes: Values expressed in mean \pm standard deviation $(n=3)$. Means in the row with different superscript are significantly different at $\mathrm{p}$ $<0.05$.

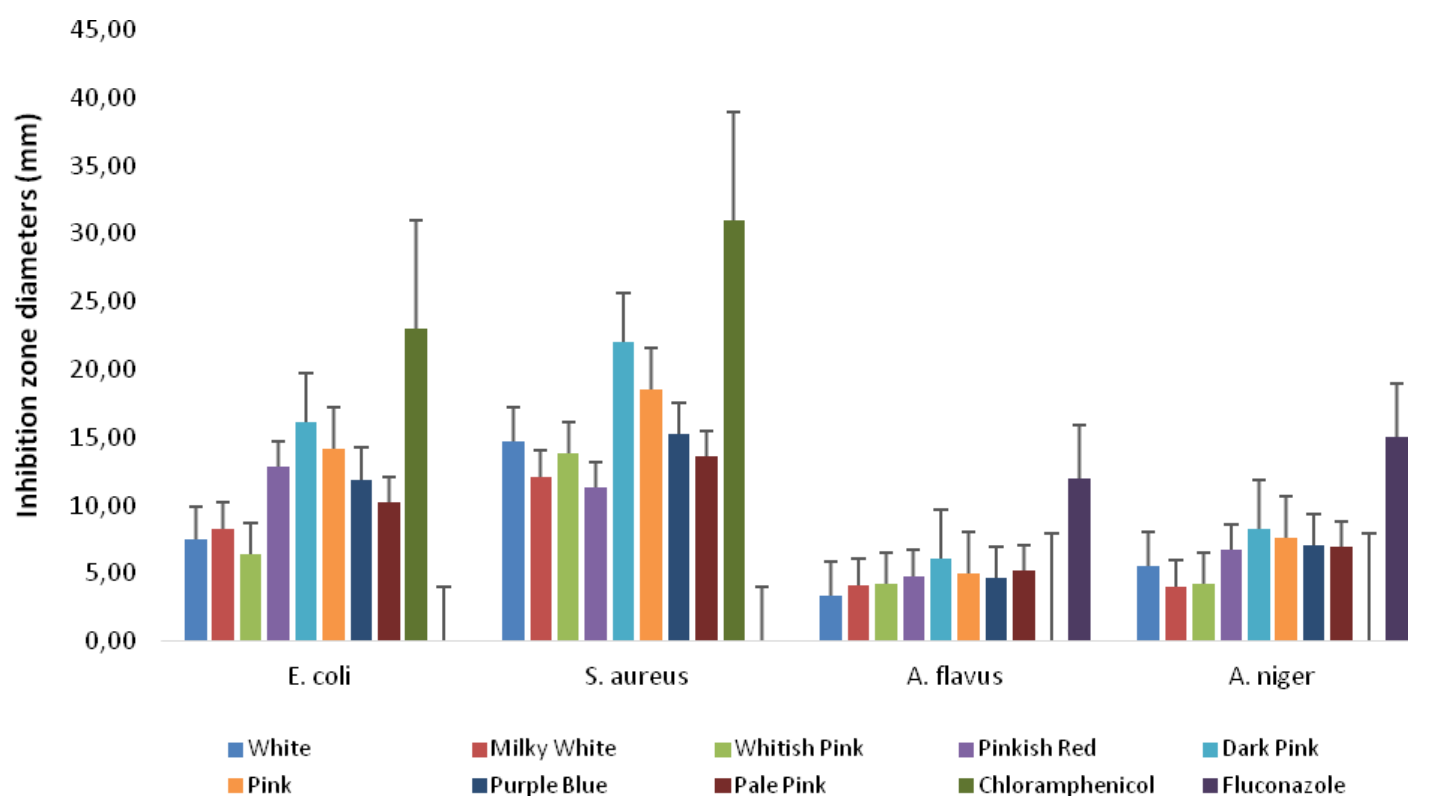

Figure 12. Results of the antimicrobial evaluation of the leaves extract showing the IZD $(\mathrm{mm})$ produced against test microbial

The methanolic extract of leaf $\mathrm{C}$. roseus cultivars by $G C$ MS analysis

The present study describes a detailed analysis of the phytoconstituents present in the methanol leaf extract of $C$. roseus cultivars (Table 4-11). Extraction is the primary step in medicinal plant study for the recovery and isolation of bioactive plant phytochemicals before analysis. A leaf is the principal organs of plant that as properties to attributed and abundance of the presence of alkaloids and phenolic compounds in C. roseus (Rani and Kapoor 2019). Balaabirami and Patharajan (2012) reported that the antimicrobial potential in crude extracts of different parts, such as leaves, stem, root, and flower of $C$. roseus against clinically significant bacterial strains. The finding shows that $C$. roseus leaf extracts have the most abundance of phytochemicals to GC-MS analysis and inhibit the growth of microbial if compared to the other part of the plant.

GC-MS analyzed the results, which include the active principles with their molecular formula, molecular weight, retention time, peak area $\%$, and composition of the bioactive components of $C$. roseus. The results of GC-MS testing indicated that $C$. roseus leaves of white and pink cultivars contained numerous bioactive phytoconstituents belonging to various classes such as tannins, glycosides, alkaloids, flavonoids, and steroids. The leaf extract quantification, by colorimetric methods, was found to be rich in phenolic compounds (Rani and Kapoor 2019).

The principle of GC-MS is separation due to differential distribution coefficients. In this chromatography, the moving phase (or mobile phase) is a carrier gas, usually an inert gas such as helium or an unreactive gas such as nitrogen. The stationary phase is a microscopic layer of liquid or polymer on an inert reliable support, inside a piece of glass or metal tubing called a column (Brun et al. 2001). The instrument used to perform gas chromatography is called a gas chromatograph (or "aero graph", "gas separator"). The gaseous compounds analyzed interact with the walls of the column, which coated with different stationary phases. This causes each compound to elute at a different time, known as the 
retention time of the compound. Secondly, the column through which the gas phase passes located in an oven where the temperature of the gas can be controlled.

In contrast, column chromatography (typically) has no such temperature control. The concentration of a compound in the gas phase is solely a function of the vapor pressure of the gas (Sermakkani and Thangapandian 2012). The source of leaves of $C$. roseus can often be identified from the peak pattern of the chromatograms obtained directly from headspace analysis. Similarly, unique qualitative and quantitative designs from a GC analysis will often help identify the source of many alcoholic beverages. The technique of fingerprint could identify false herbal products. The construction of the chromatographic fingerprints aims at evaluating the quality of herbal medicines (Kabesh et al. 2015).

The composition of the phytochemical compounds methanolic extract of leaves of eight $C$. roseus cultivars presented in Table 3. Table 4-11 presents the results of the identification of phytochemical components with retention time (RT), percentage of the area (Area \%), and a group of compounds from each cultivar of $C$. roseus. Whereas figure 3-10 is phytochemical profiles from the analysis with the GC-MS spectrum of eight $C$. roseus cultivars. Based on the results of the research, 18 components were identified (Table 3). The main components in C. roseus leaf extract were 2-Hexadecen-1-ol, 3,7,11,15-Tetramethyl-, [r- [r*, r * - (e)]] - (t-Phytol), found in var. "White" included terpenoid alcohol group $(63.26 \%)$; 2-Hexadecen-1-OL, 3,7,11,15-Tetramethyl-, [R- [R*, R* - (E)]] - (t-Phytol) in cult. "White Pink" (56.07\%) included acyclic diterpene groups; 2-Hexadecen-1-OL, 3,7,11,15-Tetramethyl-, [R- [R *, $\mathrm{R} *$ - (E)]] - (t-Phytol) in cult. "Milk-White" (54.9\%) and on the cult. "Purple Blue" (65.9\%) included diterpene alcohol; 2-Hexadecen-1-ol, 3,7,11,15-Tetramethyl-, [R- [R *, R * - (E)]] - (CAS) in cult. "Pink" (71.16\%) and on cult. "Pale Pink" (70.26\%) belongs to the aromatic compound group. Literature confirmed that the assessment of the genetic diversity of Catharanthus cultivars achieved through secondary metabolites (Saadedin 2018).

Coronaridine (R/T 10.31) in C. roseus cult. Dark Pink and Witish Pink are a member group of significant alkaloid compounds. In general, the reliability of the medicinal plant for its usage evaluated by correlating the phytochemical compounds with their biological activities Palmitic acid present in cult. Dark Pink, Purple Pink, Witish Pink, and Pinkish Red. A phytochemical compound in a group of palmitic acids, such as hexadecanoic acidmethyl ester. Hexadecanoic acid - palmitic acid (R/T 9.71) in $C$. roseus cultivars can be an antioxidant, hypocholesterolemic, nematicide, pesticide, lubricant activities and hemolytic 5-alpha is a reductase inhibitor, antiandrogenic, and flavor. Parasuraman et al. (2009) identified 17 compounds with n-hexadecanoic acid and octadecanoic acid as the major compounds in the leaves of ethyl acetate extract of Cleistanthus collinus by GC-MS analysis. C. roseus cult. White have compounds of Phytol (2-Hexadecen-1-OL,3,7,11,15-Tetramethyl) is one among the eighteen compounds of the present study. Phytol-Diterpene $(\mathrm{R} / \mathrm{T} 10.83)$ is an antimicrobial, anticancer, anti- inflammatory, and diuretic agent. Phytol-Diterpene (R/T 19.67), is an antimicrobial activity and diuretic agent of Vitex negundo leaf extract (Kumar et al. 2010). According to Hor et al. (2012), the 9,12-Octadecadienoic acid (Z,Z), also known as conjugated linoleic acid (CLA) has been demonstrated to have antimicrobial and anticancer activity. Rios et al. (2013) reported that citronellyl acetate is a monoterpene product of the secondary metabolism of plants, which has been shown in the literature to possess several different biological activities. Citronellyl Acetate (also known as citronellol acetate) is an important perfumery chemical that can be produced both by chemical catalysis and biocatalysis.

\section{Interpreting phytochemical profiles among eight $\mathrm{C}$. roseus cultivars}

The differences in phytochemical profiles considered in terms of the taxonomy and breeding history of the cultivars and possible influences from other factors such as the developmental stage of the flower. The evolutional phenotypic plasticity of the leaf accelerates plant adaptation to the environment. Because variation in phytochemistry may affect not only the evolution of the plant but also entire communities and ecosystems. Phytochemical plasticity has identified as a high priority research target (Hendry 2016). There is a tight correlation between plant metabolism and morphology, and it reported that the leaf and flower morphology could be affected by specific metabolites. The high levels of phenolic, terpenoid, linoleic acid, aromatic compound, and essential oil in all of the cultivars of $C$. roseus. Each of the plant cultivars responds to phytochemical production (Aruna et al. 2015).

A total of 18 significantly different metabolites compounds were present in different quantities. The substances represented by a homologous series of phenolic, linoleic acids, fatty acid esters, terpenoids, alkaloid, and aromatic compounds. The abundances of phytochemical compounds (18 classes total) in each cultivar were Dark Pink (66\%), Pink (50\%), Purple Pink (44\%), Pale Pink (27\%), White (44\%), Milky White (50\%), Whitish pink $(50 \%)$ and Pinkish Red (55\%) based on (Table 3, Figure 3). C. roseus cult. Dark Pink is the most abundant comparing the other cultivars. Status of this plant cultivated in the Baturraden sub-district. The Dark Pink cultivar has long been cultivated for herbal medicine and as an ornamental plant, with character white large radiating base with a yellow center. Different cultivars almost gave different compounds and different biological activities. According to Pham et al. (2017), the whole plants of White cultivar of $C$. roseus, including the flowers that have white petals and white eyes with yellow centers. The leaf and flower gave a better response to the isolation of phenolic compounds comparing the other organ.

The presence of phytochemical components such as alkaloids and flavonoids of $C$. roseus leaves are developing resistance against many of the currently available antimicrobial drugs (Patil and Ghosh 2010). Phytochemical screening of the ethanolic extract of $C$. roseus disclosed the presence of alkaloids, terpenoids, phenols, tannins, saponins, quinines, flavonoids, and proteins (Doshi et al. 
2018). C. roseus cultivar Milkey white and Pink could efficiently be used in the development of new life-saving drugs. As present findings that the organic solvent extract of $C$. roseus possesses high antimicrobial potential activity against the test organisms rather than the aqueous and crude extract. It also validates the use of these genera in an ancient medicinal system. The extract could be used in the formulation of new drugs to treat various types of infectious diseases caused by pathogens (Yadav et al. 2018). Rani and Kapoor (2019) compared the phytochemical profile and antibacterial activity of two cultivars of $C$. Roseus The results indicated that the Pink cultivar of $C$. roseus was phytochemically superior to the White cultivar.

\section{Antimicrobial properties of methanol leaf extract of $\mathrm{C}$. roseus cultivars}

Alkaloids are the most potent active chemical constituents of $C$. roseus. More than 400 alkaloids are present in the plant, which used as pharmaceuticals, agrochemicals, flavor and fragrance, ingredients, food additives, and pesticides (Satyarsa, 2019). The alkaloids like active plastidemeric, vinblastine, vincristine, vindesine, vindesine. Rosindin is an anthocyanin pigment found in the flower of $C$. roseus (Sain and Sharma 2013). The methanol extract of $C$. roseus cultivars degree of inhibitory effect against all tested microbial pathogenic strains (Table 12, Figure 12). The studies of antimicrobial property of periwinkle leaves extract have checked against microorganisms like E. coli, S. aureus. The antifungal activity has monitored against $A$. niger, A. flavus (Paikara et al. 2017).

The results Dark Pink cultivar is the optimum potential as antimicrobial activity identified with methanolic leaf extract of C. roseus cultivar against E. coli, S. aureus, A. flavus, and A. niger. Dark Pink cultivar shows the best evaluation as antimicrobial properties with a zone of inhibition $16.10 \pm 1.67 \mathrm{~mm}, 22.00 \pm 0.33 \mathrm{~mm}, 6.05 \pm 0.67 \mathrm{~mm}$, and $8.20 \pm 0.50 \mathrm{~mm}$, respectively. Following the Pinkish and Pinkish Red cultivars with inhibition zones $14.10 \pm 0.86 \mathrm{~mm}$, $18.50 \pm 0.69 \mathrm{~mm}, 5.00 \pm 0.65 \mathrm{~mm}, 7.60 \pm 0.65 \mathrm{~mm}$ and $12.80 \pm 0.61 \mathrm{~mm}, \quad 11.30 \pm 0.30 \mathrm{~mm}, 4.80 \pm 0.50 \mathrm{~mm}$, $6.70 \pm 0.45 \mathrm{~mm}$ respectively. Govindasamy and Srinivasan (2012) reported the ethanolic extract of White cultivar of $C$. roseus showed a maximum zone of inhibition against $S$. aureus is $6.24 \pm 0.69 \mathrm{~mm}$, and $5.20 \pm 0.86 \mathrm{~mm}$ zone of inhibition against $E$. coli.

Mekonnen et al. (2018) reported that the antibacterial mechanism of metabolite compound includes denaturation of bacterial protein, disruption of the bacterial cell membrane, and inhibition of nucleic acid synthesis process will cause DNA damage, thus disturbing bacterial growth. Antibacterial properties of $C$. roseus extract could break down bacterial cell walls, thus inhibiting the growth of bacteria E. coli and S. aureus. Paikara et al. (2017), C. roseus leaves extract consisting of alkaloids, which have antimicrobial activity through the mechanism of structural changes and the overhaul of the structure of amino acids in the body of microbes. As a result of this, a clear zone was forming as an antimicrobial parameter.
The efficiency of the active composites causes the creation of growth inhibition zones that appear as clear areas near the disk. In this research, the methanolic extract of eight leaves extract of $C$. roseus cultivars showed potent antimicrobial activity against these microorganisms. The variation in the effectiveness of leaves extract against different strains could depend on the differences in the permeability of cells of those microbes. High antimicrobial activity was demonstrated in the extracts of leave and suggested that they may be useful in the treatment of infections caused by microorganisms.

The presently studied $C$. roseus plant extract could be of considerable infers to the development of new lifesaving drugs. However, further research is required to isolate the bioactive principle of this species as well as further studies on its bio efficiency against human pathogens. It can conclude from the present findings that the ethanolic extract of $C$. roseus collected from the Banyumas region was showed potential antimicrobial activity source for various infects. Further, studies need to conform to identify the particular compounds to use as a drug as the main ingredient in traditional medicine.

The novelty of this study is to describe the results of the exploration of eight different $C$. roseus cultivars. Next, conduct a GC-MS test to determine the diversity of phytochemicals at the cultivar level and to know the antimicrobial ability between cultivars. Most previous studies or other studies did not describe in detail the capabilities of $C$. roseus cultivars, only to mention $C$. roseus in general.

Analysis using GC-MS of methanol extract of C. roseus cultivars was obtained 18 phytochemical components identified. The investigation revealed the presence of hydrocarbon alkane, phenolic, linoleic acids, fatty acid esters, terpenoids, alkaloid, aromatic compounds, and fatty acids in 8 cultivars of $C$. roseus. The Dark Pink cultivar has the most abundant phytochemical compounds compared to other cultivars. C. roseus cult. Dark Pink gave the best antimicrobial activity against E. coli, S. aureus, A. flavus, and $A$. niger with the zone of inhibition $16.10 \pm 1.67 \mathrm{~mm}$, $22.00 \pm 0.33 \mathrm{~mm}, \quad 6.05 \pm 0.67 \mathrm{~mm}$ and $8.20 \pm 0.50 \mathrm{~mm}$ respectively. Different cultivars gave different responses to the phytochemical content and antimicrobial activity, but not correlated with morphology of corolla color. The importance of the research is due to the basic compounds of antimicrobial activity. This research, which reveals the presence of components in $C$. roseus cultivars, suggests that the contribution of these compounds on the pharmacological activity should be evaluated.

\section{ACKNOWLEDGEMENTS}

This work was carried out with the support of Research and Public Service Institute of Jenderal Soedirman University for Competency Research Scheme of Financial Year 2019: No. DIPA P/359/UN 23/14/PM/2019. Many thanks to Deska for helping the chromatographic process. We also thank the farmers of medicinal plants in the Banyumas District who helped provide the sample material. 


\section{REFERENCES}

Adnan KN, Saenab S. 2016. Utilization of Catharanthus roseus as a senior high school and biology science learning resource. J Bionat 17 (2): 97-101.

Almagro L, Fernández-Pérez F, Pedreño MA. 2015. Indole alkaloids from Catharanthus roseus: bioproduction and their effect on human health. Molecules 20 (2): 2973-3000.

Aruna MS, Prabha MS, Priya NS, Nadendla R. 2015. Catharanthus roseus: the ornamental plant is now medicinal boutique. J Drug Delivery Ther 5: 1-4.

Atef NM, Shanab SM, Negm SI, Abbas YA. 2019. Evaluation of antimicrobial activity of some plant extracts against antibiotic susceptible and resistant bacterial strains causing wound infection. Bull Nat Res Cen 43 (1): 144-149.

Balaabirami S, Patharajan S. 2012. In vitro antimicrobial and antifungal activity of Catharanthus roseus leaves extract against important pathogenic organisms. Intl J Pharm Pharm Sci 4 (3): 487-490.

Brun G, Bessiere GM, Genevieve M, Dijoux-Franca MG, David B, Mariotte AM. 2001. Volatile components of Catharanthus roseus (L.) G. Don (Apocynaceae). Flavour Fragr. J. 16: 116-119.

Chaman S, Garima S, Shalini, Anil KR. 2013. Study of Antimicrobial properties of Catharanthus roseus by agar well diffusion method. Int Res J Pharm Appl Sci 3 (5):65-68.

Chen Q, Lu X, Guo X, Guo Q, Li D. 2017. Metabolomics characterization of two Apocynaceae plants, Catharanthus roseus and Vinca minor, using GC-MS and LC-MS methods in combination. Molecules 22 (6): 997.

Davis W, Stout TR. 1971. Disc plate method of microbiological antibiotic assay. Am Soc Microbiol 22 (4): 659-665.

Doshi GM, Matthews BD, Chaskar PK. 2018. Gas Chromatography Mass Spectroscopy studies on ethanolic extract of dried leaves of Catharanthus roseus. Asian J Pharm Clin Res 11 (6): 336-340.

El-Domyati FM, Ramadan AM, Gadalla NO, Edris S, Shokry AM, Hassan SM, Abuzinadah OA. 2012. Identification of molecular markers for flower characteristics in Catharanthus roseus producing anticancer compounds. Life Sci J 9 (4): 5949-5960.

Gajalakshmi S, Vijayalakshmi S, Rajeswari V. 2013. Pharmacological Activities of Catharanthus roseus. Intl J Pharma Bio Sci 4 (2): 431-439.

Hendry AP. 2016. Key questions on the role of phenotypic plasticity in eco-evolutionary dynamics. J Heredity 107 (1): 25-41.

Hor SY, Ahmad M, Farsi E, Yam M F, Hashim MA, Lim CP, Asmawi M Z. 2012. Safety assessment of methanol extract of red dragon fruit (Hylocereus polyrhizus): Acute and subchronic toxicity studies. Regul Toxicol Pharmacol 63 (1): 106-114.

Hussein HJ, Hameed IH, Hadi MY. 2017. Using Gas ChromatographyMass Spectrometry (GC-MS) technique for analysis of bioactive compounds of methanolic leaves extract of Lepidium sativum. Res J Pharm Tech 10 (11): 3981-3889.

Jacobs DI, Snoeijer W, Hallard D, Verpoorte R. 2004. The Catharanthus alkaloids: Pharmacognosy and biotechnology. Curr Med Chem 11: 607-628.

Kabesh K, Senthilkumar P, Ragunathan R, Kumar RR. 2015 Phytochemical analysis of Catharanthus roseus plant extract and its antimicrobial activity. Intl J Pure Appl Biosci 3 (2): 162-172.

Kar GA, Rout S K, Mishra D. 2018. Phytochemical screening and GC-MS analysis of methanol extract of the leaves of Ipomoea carnea. World J Pharmaceut Res 7 (13): 887-895.

Karthikeyan K, Dhanapal CK. 2016. GC-MS analysis of methanol extract of Alysicarpus monilifer-whole plant. Der Pharma Chemica 8 (19) 563-571.

Karthishwaran K, Muthukkumarasamy S, Sankaran M. 2012. GCMS analysis of methanolic extract of aerial parts of Pergularia daemia. J Life Sci 1 (1): 50-55.

Koul M, Lakra NS, Chandra R, Chandra S. 2013. Catharanthus roseus and prospects of its endophytes: a new avenue for production of bioactive metabolites. Intl J Pharmaceut Sci Res 4 (7): 2705-2716.

Kumar PP, Kumaravel S, Lalitha C. 2010. Screening of antioxidant activity, total phenolics and GC-MS study of Vitex negundo. Afr J Biochem Res 4 (7): 191-195.

Lal S, Mistry KN, Shah SD, Thaker R, Vaidya PB. 2011. Genetic diversity assessment in nine cultivars of Catharanthus roseus from Central Gujarat (India) through RAPD, ISSR and SSR markers. J Biol Res 1 (8): 667-675.

Mekonnen SA, Lam TJGM, Hoekstra J, Rutten VP, Tessema TS, Broens EM. 2018. Characterization of Staphylococcus aureus isolated from milk samples ofdairy cows in small holder farms of North-Western Ethiopia. BMC Vet Res 14: 246-250.

Mohan SCT, Priyadarshini GS, Balamurugan V. 2015. GC-MS analysis of phytochemicals and hypoglycemic effect of Catharathus roseus in alloxan induced diabetic rats. Intl J Pharm Sci Res 25: 123-128.

Muthulakshmi A, Jothibai MR, Mohan VR. 2012. GC-MS analysis of bioactive components of Feronia elephantum correa (Rutaceae). J Appl Pharm Sci 2 (2): 69-74.

Nayak BS, Pereira LMP. 2006. Catharanthus roseus flower extract has wound-healing activity in Sprague Dawley rats. BMC Compl Altern Med 6 (1): 41. DOI: 10.1186/1472-6882-6-41

Paikara DIVYA, Pandey B, Singh SHEETAL. 2017. Phytochemical analysis and antimicrobial activity of Catharanthus roseus. Indian $\mathrm{J}$ Sci Res 12 (2): 124-127.

Painuli S, Rai N, Kumar N. 2016. Gas chromatography and mass spectrometry analysis of methanolic extract of leaves of Rhododendron arboreum. Asian J Pharm Clin Res 9: 101-104.

Pandiangan D, Tilaar W, Nainggolan N, Wahyudi L. 2013. Relations between catharanthine content enhancement with the other associated secondary metabolites in Catharanthus roseus cell culture that treated tryptophan. Intl J Sci Res 4 (1): 2208-2212.

Parasuraman S, Raveendran R, Madhavrao C. 2009. GC-MS analysis of leaf extracts of Cleistanthus collinus Roxb. (Euphorbiaceae). Int. J Ph Sci 1 (2): 284-286.

Patil PJ, Ghosh JS. 2010. Antimicrobial activity of Catharanthus roseus-a detailed study. British J Pharmacol Toxicol 1 (1): 40-44.

Pereira DM, Faria J, Gaspar L, Ferreres F, Valentao P, Sottomayor M, Andrade PB. 2010. Exploiting Catharanthus roseus roots: Source of antioxidants. Food Chem 121: 56-61.

Pham HNT, Vuong QV, Bowyer MC, Scarlett CJ. 2017. Effect of extraction solvents and thermal drying methods on bioactive compounds and antioxidant properties of Catharanthus roseus (L.) G. Don (Patricia White cultivar). J Food Process Preserv 41 (5): 285-301.

Pinho PGD, Goncalves RF, Valentão P, Pereira DM, Seabra RM, Andrade PB, Sottomayor M. 2009. Volatile Composition of Catharanthus roseus (L.) G. Don Using Solid-phase Microextraction and Gas Chromatography/Mass Spectrometry. J Pharmaceut Biomed Anal 49: 674-685.

Rani J, Kapoor M. 2019. Gas chromatography-mass spectrometric analysis and identification of bioactive constituents of Catharanthus roseus and its antioxidant activity. Asian J Pharm Clin Res 12 (3): 461-465.

Rios ERV, Rocha NFM, Carvalho AMR, Vasconcelos L F, Dias ML, de Sousa DP, de França Fonteles MM. 2013. TRP and ASIC channels mediate the antinociceptive effect of citronellyl acetate. Chem Biol Infect 203 (3): 573-579.

Saadedin SM. 2018. Genetic diversity and alkaloids profile evaluation of Catharanthus roseus L based on RAPD molecular markers and RPHPLC analysis. Pak J Biotechnol 15 (2): 541-551.

Sain M, Sharma V. 2013. Catharanthus roseus (an anti-cancerous drug yielding plant). A review of potential therapeutic properties. Intl J Pure App Biosci 1 (6): 139-142.

Samiyarsih S, Naipospos N, Palupi D. 2019. Variability of Catharanthus roseus based on morphological and anatomical characters, and chlorophyll contents. Biodiversitas 20 (10): 2986-2993.

Sasongko ND, Samiyarsih S. 2018. Half seed chromatography-a non destructive method in seed's mass selection. Prosiding: Pengembangan Sumber Daya Perdesaan dan Kearifan Lokal Berkelanjutan VIII, 14-15 November 2018 Purwokerto 8 (1): 111122. [Indonesian]

Satyarsa ABS. 2019. Potential effects of alkaloid vindolicine substances in tapak dara leafs (Catharanthus roseus (L.) G. Don) in reducing blood glucose levels. J Med Health 2 (4): 1009-1019.

Sermakkani M, Thangapandian V. 2012. GC-MS analysis of Cassia italica leaf methanol extract. Asian J Pharm Clin Res 5 (2): 90-94.

Shimadzu. 2008. GCMS-QP 2010 Plus. http://www.shimadzu.com. [16 Sep 2008].

Vanitha V, Umadevi KJ, Vijayalakshmi K. 2011. Determination of bioactive components of Annona squamosa L leaf by GC-MS analysis. Intl J Pharm Sci Drug Res 3 (4): 309-312.

Velmurugan G, Anand SP. 2017. GC-MS analysis of bioactive compounds on ethanolic leaf extract of Phyllodium pulchellum L. Intl J Pharm Phytochem Res 9 (1): 114-118.

Yadav AK, Ambasta SK, Prasad SK, Trvedi MP. 2018. In-vitro evaluation of antibacterial property of Catharanthus roseus (Linn.) G. Don. Var."rosea" and "alba”. Intl J Pharm Pharm Sci 10: 55:58. 
BIODIVERSITAS

Volume 21, Number 4, April 2020

Pages: 1332-1344
ISSN: 1412-033X

E-ISSN: 2085-4722

DOI: 10.13057/biodiv/d210409 